reading this monograph is greatly facilitated by the excellent way in which the volume is produced and illustrated, including some electron micrographs vividly illustrating the cellular components.

The only criticisms I can make of this publication are those which apply in general to a collection of review articles of this kind written by different authors and dealing with various aspects of research on a particular topic. In the first place it is always extremely difficult under these circumstances to avoid repetition of material. For example, in the present monograph the work which led to the recognition of the brown adipose tissue as being a major site of heat production is referred to a number of times, and likewise a description of the microscopic anatomy of the tissue is given by several authors. Secondly, there is, in some respects, a lack of integration in the separate articles dealing with closely related topics as, for example, the last four chapters dealing with the fascinating problems of the mitochondrial activity of this tissue and the special mechanisms by which the energy released in substrate oxidation is converted into heat and not stored as high energy phosphate bonds in ATP as in other tissues. In my opinion this general topic could, perhaps, have been better covered in one single chapter from which a clearer picture of the present state of knowledge of this complex field (which is obviously still very incomplete and the conclusions are, in some respects, controversial) could be obtained.

For a biochemist with physiological interests it is reassuring to be aware that there are still many investigators actively engaged on research into biochemical mechanisms in relation to physiological function; the emphasis today in the biochemical literature is on studies on isolated systems unrelated not only to intact organisms but even into intact cells, and for this reason I found reading this monograph a stimulating and welcome change.

ANNE Beloff-Chain

\section{Invertebrates in vitro}

Invertebrate Organ Cultures: Colloquium on Experimental Embryology, Clermont-Ferrand, April 1968. (Documents on Biology, No. 2.) Organized by H. Lutz. Pp. xiv +252. (Gordon and Breach: New York and London, December 1970.) \$22, £9.25 boards; $\$ 7.50$, £3.15 paper.

This book is the second in a series designed to provide review articles for students and their teachers. It contains papers originally read at a conference held in 1968, most, but not all, of which deal with morphogenesis and cell differentiation in organ culture. It would be wrong to expect a very coherent picture to emerge. Insects, molluscs, echinoderms, crustaceans and coelenterates have invited different kinds of question and present different obstacles to enquiry. It is a pity, but no fault of the authors, that the common interest striking the reader most forcibly is a technical one. Of some of the articles it would certainly be fair to say that the culture medium is the message. This is above all true of the major technical survey of methods by $\mathrm{N}$. Le Douarin.

Nevertheless, it is clear that invertebrate organ culture, which has in the past lagged behind work with birds and mammals, is at the beginning of a very promising period. For coelenterates and planarians (here treated by $L$. Gomot and C. Ziller-Sengel) it can contribute to morphogenetic problems that now have classical status-metaplasia in the hydroid and determination of regeneration blastemata in the flatworm. In other invertebrates culture work is particularly helpful in clarifying the endocrine control of morphogenesis. The articles by L. Gomot and by $P$. Lubet and W. Streiff establish this for molluscs. Insects are treated by T. Lender and by P. Nardon and $G$. Plantevin, crustaceans by J. BerreurBonnenfant and echinoderms by $\mathbf{R}$. Delavault and J. Bruslé.

As its editors intend, this volume will be of real value to readers not already involved in the field. D. R. NEWTH

\section{Chemical Triggers}

Chemicals Controlling Insect Behavior. By Morton Beroza. Pp. xii +170. (Academic: New York and London, October 1970.) $£ 4.65$.

THE six articles by sixteen authors published in this book were first presented in 1969 at an American Chemical Society Symposium of the same title. Two other books, Control of Insect Behavior by Natural Products edited by David L. Wood, Robert M. Silverstein and Minoura Nakajima, and Chemical Ecology edited by E. Sondheimer and John B. Simeone, also published by Academic Press in 1970, cover the same ground somewhat more comprehensively. All three books reflect an awareness on the part of ruany biologists and chemists in the United States, Japan and Canada of the need to find safer and more efficient alternatives to the broad spectrum pesticides still in use.

Although the importance and potential economic application of naturally occurring chemicals, including pheromones and allomones, for controlling the spatial and temporal structure of populations have long been realized by some ecologists, the progress of this research field has been slow. The pace has accelerated now that the importance of an interdisciplinary approach, involving chemists and biologists, has been realized, and made more attractive by the recent technological advances which permit the rapid characterization and purification of natural products in micro quantities. This book does not attempt to give a comprehensive coverage of the subject matter, and as there has been no serious attempt at an integrated approach it loses some of its value. Not unexpectedly, the emphasis is on chemistry rather than on behaviour. The first chapter deals with sex pheromones in the Lepidoptera, the second with the so-called sex pheromones of the beetle Ips confusus, and the third with the sex attractants of the boll weevil Anthonomus grandis. The factor which attracts Ips confusus is described as a pheromone despite the fact that it is diet dependent and that its site of production has not yet been identified. It is evident that difficulties were encountered in the behavioural assays because of synergistic and antagonistic effects, and that there were problems of standardizing stimulus application, the receptor animals and the response. The chemistry is at a more sophisticated level than the behavioural work, and a great deal more work on behaviour needs to be done to explain, for example, why one pheromone is a powerful male stimulant in the laboratory but not a long range attractant in the field.

Professor Blum's review of the pheromones of social insects underlines the fact that a great deal more is known about these pheromones and their mode of action both as primers and releasers. It might be suggested that investigations into the primer effects of pheromones in non-social insects might repay study. The fifth chapter gives a good comprehensive review of the biochemistry of arthopod defensive secretions, but as the behavioural content is minimal the link with the title of the symposium is somewhat tenuous. The last chapter, by Dr Beroza, succinctly summarizes recent development and current usage of insect attractants and repellents in the United States Department of Agriculture. The support that this work on attractants gives to the stereochemical theory of olfaction is discussed briefly. The thesis that "the greater the number of chemicals tested the better the chance of finding a potent attractant" could be challenged. An indiscriminate search for attractants might well be wasteful of resources, and behavioural studies carried out on the responses of animals to chemicals in their natural environments might be more profitable. The layout, photo- 